Of course, the aetiology of anorexia nervosa is multi-factorial, but to suggest that difficulties in family functioning do not cause the condition, is surely as inaccurate as to say that the condition is not caused by genetic factors, life events or societal pressures to be thin. All these factors are likely to play a part, to a greater or lesser extent, in an individual case.

S. G. Gowers Academic Unit, Young People's Centre, Pine Lodge, 79 Liverpool Road, Chester $\mathrm{CH} 2$ IAW

C. North North Essex Child \& Family Consultation Service, Clacton-on-Sea, Essex

\section{Suicide attempts v. deliberate self-harm}

Isometsä \& Lönnqvist (1998), who focus on a clinical variable of deliberate self-harm for predicting future repetition or eventual suicide, only touched upon the issue of the ambiguity in their definition of suicide attempts/deliberate self-harm. The authors contend that this ambiguity in definition does not introduce "any bias in the reporting of previous attempts".

Unfortunately, any ambiguity in a definition always has the potential to introduce bias in epidemiological studies and this is why operating definitions of caseness are so important. Stating their operational definition could have removed the ambiguity and the potential for bias.

It is important to recognise the two syndromes of self-harm, namely suicide attempt (failed suicide) and non-fatal deliberate self-harm when death was not intended, sometimes pejoratively referred to as parasuicide, but which term remains useful. In the former syndrome, the act is designed to be fatal and any survival is accidental. In the latter, the act is designed not to be fatal and so any death is accidental. Deliberate self-harm is more common in females, although differences in gender-specific rates are narrowing (Hawton et al, 1997). It often leads to frustration among health care workers and deterioration in their relationship with the patient, with the potential for developing "malignant alienation" (Watts \& Morgan, 1994). Any approach to secondary prevention of suicide must recognise this distinction, although the attitude towards the latter syndrome must be serious, sensitive and sympathetic. Unless the definition of caseness of 'suicide attempt' in this study was clearly stated to exclude the second group of subjects, there could be a serious bias to the study. This could account for the higher proportion of female suicides who were not first-time attempters. The bias in the study would be that the finding that most male completed suicides die in their first suicide attempt may be a spurious one resulting from the lack of operational definition of suicide attempt and the inclusion of 'parasuicide' in the study.

Also, the authors have not indicated whether pregnant women and postnatal women have been excluded from the study. Pregnancy and motherhood have been postulated to provide some protection against suicide (Appleby, 1991).

The implication is that the major conclusion of the study - that the approach to suicide prevention should be different for men and women - may be erroneous.

Appleby, L. (1991) Suicide during pregnancy and in the first postnatal year. British Medicol Journal, 302. 137-140.

Hawton, K., Fagz, J., Simkin, S., et al (1997) Trends in deliberate self-harm in Oxford, 1985-1995. Implications for clinical services and prevention of suicide. British Journal of Psychiotry, I7I, 556-560.

Lometsä, E.T. \& Lönnquist, J. K. (1998) Suicide attempts preceding completed suicide. British journal of Psychiotry, 173, 531-535.

Watts, D. Morgan, G. (1994) Malignant alienation: Dangers for patients who are hard to like. British journa of Psychiotry, 164, 11-15.

L. O. Ogundipe Edward Myers Addiction Unit, City General Hospital, Stoke-on-Trent ST4 6QG

Authors' reply: Dr Ogundipe has given us the opportunity to clarify several points. His main argument was that our definition of a suicide attempt was ambiguous and did not exclude parasuicides (as opposed to true suicide attempts), which resulted in a bias in our comparison between genders, as more females conduct parasuicidal acts than males; we disagree.

The definition for a suicide attempt in the research phase of the National Suicide Prevention Project in Finland was a clinical one, based on the field researcher's (a mental health professional) evaluation of the clinical significance of the act. Some degree of suicide intention needed to be present in order to rate an act as a suicide attempt, and the act needed to be fully undertaken, not only planned or aborted. We do not share Dr Ogundipe's conviction that suicidal acts can be reliably classified into 'suicide attempts' and 'parasuicides'. Studies of suicide intent among suicide attempters/ parasuicides do not show a bimodal distribution of suicide intent (Suominen et al, 1997). In the pan-European WHO/Euro Multicentre Study on Parasuicide, the concepts of 'suicide attempt' and 'parasuicide' were used interchangeably (Ostamo \& Lönnqvist, 1994).

Contrary to what Dr Ogundipe expects, the higher rate of parasuicide among females is not true in Finland. In fact, Finland is the only country in Europe where males seem to have a slightly higher incidence of parasuicides than females (Ostamo \& Lönnqvist, 1994).

Our overall prevalence of suicides with previous attempts (44\%) in Finland was generally in line with the five earlier, smaller studies we referred to $(21-32 \%)$. As the mean age of suicides in Finland was somewhat lower, and preceding attempts are more prevalent in the younger age groups, the minor difference would likely totally disappear if the data were age-adjusted before comparisons. Finally, we would like to stress that we did not claim that suicide prevention needs to be totally different for males or females. On the grounds of our nationwide data, we pointed out the importance of early intervention, particularly (but not exclusively) among males.

Ostamo, A. \& Lönnquist, J. (1994) The epidemiology of attempted suicide in Helsinki, Finland 1989-1993. In Attempted Suicide in Europe. Findings from the Multicentre Study on Parosuicide by the WHO Regional Office for Europe (eds A. J. F. M. Kerkhof, A. Schmidtke, U. Bille-Brahe, et al). pp. 137-158. Leiden: DSWO Press.

Suominen, K., lsometeia, E., Henrikseon, M., et at (1997) Differences in hopelessness, suicide intent and impulsivity among suicide attempters with major depression, alcohol dependence or both. Acto Psychiatrica Scandinovico, 96, 142-149.

E. lsometsä, J. Lönnqvist Department of Mental Health and Alcohol Research, National Public Health Institute, Helsinki, Finland

\section{Lithium and suicide prevention}

Sir: Isacsson et al (1999) recommended that highly suicidal patients should be referred to psychiatric hospitals for supervision and often electroconvulsive therapy (ECT). We would like to draw attention to observations that might contribute to the prevention of suicides in highly suicidal patients. 